East African Medical Journal Vol. 80 No.7 July 2003

MEASLES TRENDS AND VACCINE EFFECTIVENESS IN NAIROBI, KENYA

P. K. Borus DrPH,Senior Research Officer, Centre for Virus Research, Kenya Medical Research Institute, P. Cumberland MSc., Lecturer, London School of Hygiene and Tropical Medicine, S. Sonoiya MMed, Manager, Kenya Expanded Programme on Immunisation, J. Kombich MSc, Assistant Research Officer, P.M. Tukei MD., Chief Research Officer, Centre for Virus Research, Kenya Medical Research Institute and F.T. Cutts MD, Professor of International Health, Medical Research Council, Gambia

Request for reprints to: Dr. P. Borus, Centre for Virus Research, Kenya Medical Research Institute, P.O. Box 54628, Nairobi, Kenya

\title{
MEASLES TRENDS AND VACCINE EFFECTIVENESS IN NAIROBI, KENYA
}

\section{P. K. BORUS, P. CUMBERLAND, S. SONOIYA, J. KOMBICH, P.M. TUKEI and F.T. CUTTS}

\begin{abstract}
Objectives: To determine morbidity and mortality from measles and to estimate measles vaccine effectiveness among children hospitalised with measles in two hospitals in Nairobi. Design: A review of hospital records (index cards).

Setting: Kenyatta National Hospital and Mbagathi District Hospitals covering the years 1996-2000.

Method: A review of index cards for measles morbility and mortality was undertaken in the two hospitals. Measles data at the Kenya Expanded Programme on Immunisation covering both hospitals was analysed for vaccine effectiveness.

Results: The incidence of measles was unusually high in 1998 between July and November (monthly range 130-305), reflecting on the occurrence of an outbreak at that time. There was no definite monthly incidence trend of measles in 1996, 1997, 1999 and 2000. The median age of cases was 13 months (range 0-420 months) for Kenyatta hospital and 18 months (range 1336 months) for Mbagathi Hospital. Significantly, 29.8\% of all cases were aged below nine months when routine immunisation for measles had not begun. The median number of days spent in hospital were five days (range 0-87 days) for Kenyatta and four days (range 1-13 days) for Mbagathi. The overall case fatality rate was $\mathbf{5 . 6 \%}$ and was similar for both males and females. The overall measles vaccine effectiveness among measles cases admitted to Kenyatta and Mbagathi Hospitals was 84.1\%.

Conclusion: The case admissions in Kenyatta and Mbagathi Hospitals suggest measles was prevalent in Nairobi over the latter half decade of the 1990's. Apart from 1998 when there was an outbreak, the seasonality of measles was dampened. The 1998 outbreak suggests a build up of susceptible children the majority of whom were born in the last quarter of 1996. The high mortality may have had to do with the majority of cases presenting late when symptoms were already complicated and severe.
\end{abstract}

\section{INTRODUCTION}

Over the last decade, measles continued to be a significant cause of childhood morbidity and mortality despite widespread use of measles vaccine. In 1996, the reported number of measles cases was 0.8 million, but due to under-reporting the actual global morbidity and mortality were estimated to be 36.5 million cases and 1 million deaths respectively (1). The year 2000 mortality estimate attributable to measles was 777,000 deaths (2). This represents $10 \%$ of mortality by cause attributed to infectious diseases in children under the age of five years, and accounts for about half of annual deaths due to childhood vaccine preventable diseases. It is estimated that 11 million measles cases and half million deaths occur annually in Africa.

Measles incidence in many countries may be explained by the changing epidemiology of measles following vaccination as predicted in mathematical models (3). Early in the last decade some authors pointed out an age shift in measles incidence to older susceptible groups not covered by routine immunisation in spite of high reported vaccine coverage $(3,4)$. "Post-honeymoon" outbreaks may also occur after the period of low measles incidence when children who were missed in birth cohorts targeted during immunisation accumulate and will neither have protective antibody as a result of vaccine or from natural infection $(3,5)$.

In Kenya, fairly high levels of measles immunisation coverage were achieved over the last decade. Recent measles outbreaks are raising questions about the extent of immunisation reached as well as actual vaccine effectiveness. Vaccine effectiveness is the percent reduction in disease incidence attributable to vaccination. It is a field measure of how efficacious a vaccine is and may be influenced by determinants such as the status of the cold chain as well as immunisation practises. It's measurement provides an important epidemiological tool for monitoring the impact of immunisation programmes (6). Studies on vaccine effectiveness compare the risk of disease in vaccinated and unvaccinated groups (7). Community cohort and case control studies have been used to measure vaccine effectiveness where the likelyhood of detecting cases among immunised and non-immunised individuals should ideally be the same. However, when 
only information on cases is available, the screening method may be used to determine vaccine effectiveness (8). This has been applied in routine monitoring of vaccine coverage particularly where there are periodic large national surveys or in systems where vaccination records have been computerised (9-11). As with other methods, it compares the proportion vaccinated among cases and the population, but is controlled by external standardisation using an estimate of vaccine coverage.

Published studies in Kenya documenting vaccine effectiveness are limited in number. A study in 1988 in a rural area reported measles vaccine effectiveness of $62 \%$ among 252 cases (12). During an outbreak investigation in the lake shore district of Siaya, vaccine effectiveness was even lower at $18 \%$ while in Kirinyaga district of Central province, vaccine effectiveness was $43.5 \%$ for children between eight months and five years $(13,14)$.

This study is a review of hospital records in two government hospitals in Nairobi for reported measles disease burden. The main aim was to analyse morbidity and mortality trends over five years in the last decade. Kenyatta National Hospital (KNH) and Mbagathi hospital were chosen for the reviews because they are big government hospitals where a large number of measles cases in Nairobi would be admitted as a result of the minimal user fees they levy for paediatric services.

\section{MATERIALS AND METHODS}

The review in Kenyatta and Mbagathi hospitals only covered in-patients. All measles inpatient records were included in the study, but during analyses of vaccine effectiveness, only records of children between 0-5 years were included. Kenyatta hospital records on admissions were organised by disease, including measles, and year on index cards. A single card consisted of summaries from several cases. Each card had information on hospital number, name, sex, date of birth, outcome and number of in-patient days. However, there was no information on vaccination status.

Permission to carry out the hospital records review was obtained from the director and hospital ethical committee of $\mathrm{KNH}$ and the hospital superintendent and management of Mbagathi hospital. The information was transcribed from cards to a simple form and then entered into EPI INFO. Analysis was carried out using STATA Software (15). Data on measles morbidity from Kenyatta and Mbagathi hospitals, covering the years 1997 to 2000, was obtained from KEPI. This data was compiled from individual patient records. Measles vaccination status as recorded in individual patient records in the hospital was present for most of these. This was either from documented evidence of vaccination from a child health card or from history of vaccination. This data set was used only to estimate vaccine effectiveness. Vaccine effectiveness, the percent reduction in measles incidence attributed to vaccination was calculated for the KEPI data set using the screening method (8). The formula used was; vaccine effectiveness $(\mathrm{VE})=1-(\mathrm{Pv} / 1-\mathrm{pv})(1-\mathrm{C}) / \mathrm{C})$ where:

$\mathrm{Pv}=$ Proportion of cases vaccinated

$\mathrm{C}=$ Proportion of population vaccinated (coverage)

\section{RESULTS}

One thousand two hundred measles cases were reviewed from both hospitals. Nine hundred and forty three were from Kenyatta while 257 were from Mbagathi. Kenyatta had similar number of male and female cases, while Mbagathi had 44\% females and $56 \%$ males.

\section{Figure 1}

Variation in measles incidence

Figure 1 shows the trend of cases with time from 1996 to 2000. There were peaking of incidence in 1998 when Kenyatta hospital had more than 709 cases and Mbagathi 150 cases.

In 1998, incidence was unusually high between July and November (monthly range 130-305), reflecting on the occurrence of an outbreak at this time (Figure 2).

\section{Figure 2}

Variation of measles cases by month in 1998

The peak was in October when 305 cases were recorded (Figure 2). There was no definite monthly trend in incidence of measles in 1996, 1997, 1999 and 2000. The median age of cases was 13 and 18 months for Kenyatta and Mbagathi hospital respectively. The median ages in 1996, 1997, 1998, 1999 and 2000 were 12, 18, 13, 20 and 11 months respectively. 
Ninety per cent of cases were less than six years old while $75 \%$ were three years or younger. Significantly, in Kenyatta hospital, $25 \%$ of cases were below the age of nine months when measles vaccine is not routinely given. The median number of days spent in hospital were five days for Kenyatta and four days for Mbagathi. The overall case fatality rate was $5.63 \%$ and was similar for both males and females. There were variations in case fatality rates by year. It was $13.4 \%, 1.7 \%, 6.6 \%, 7.4 \%$ and $0 \%$ for 1996 , 1997, 1998, 1999 and 2000 respectively.

The number of cases in the KEPI data who were vaccinated was $554(38.9 \%)$ whereas $872(61.2 \%)$ cases were not. The overall vaccine effectiveness among measles cases admitted to Kenyatta and Mbagathi hospitals was, therefore, determined to be $84.1 \%$.

\section{DISCUSSION}

The cases reviewed in Kenyatta and Mbagathi hospitals suggest measles was prevalent in Nairobi over the last half decade of the 1990's. Apart from 1998 when there was an outbreak, the seasonality of measles was dampened by immunisation as in previous studies where vaccine coverage was high (16). During the 1998 outbreak, a distinct seasonal occurrence of measles was observed with peak incidence in the third quarter. Similar seasonality trends occurred in Nairobi during the pre-EPI era, when measles was reported to peak between June and November when the weather is cold and damp (17).

The proportion of children who had measles before the age for routine immunisation was $25 \%$ and there was no significant yearly variation in this proportion. This compares to earlier findings in Kinshasa by Taylor and colleagues who found $27 \%$ of infants contracted measles before nine months of age (18). It also compares well with other findings on mortality from measles in infancy $(4,17,19,20)$. This revisits policy concerns as to whether an earlier dose of measles vaccine could be given at six months and a second dose at nine months in some particularly densely populated areas of the major cities where measles is bound to be a problem.

The median ages of cases suggest the majority of them in 1996 and 1997 were born in 1995 and cases in 1998 were born towards the end of 1996 . The 1998 outbreak suggests a build up of susceptible children, the majority of whom were born in the last quarter of 1996. Tied to the observation that cases occurring in 1996 and 1997 were born in 1995, this point to lapses in the performance of KEPI, after the withdrawal of DANIDA in 1994 affected vaccine supply and programme function. The 1998 outbreak could also signify the contribution of potential pockets of low immunisation coverage as seen in the resurgence of measles in urban areas with good general coverage (21).

Vaccine effectiveness in this study was determined using the screening method. Although its value was fairly high, there may be some bias in this estimate resulting from inaccuracies in the value of vaccination coverage, derived as an average estimate by KEPI over the last decade, used in this study. A real vaccine coverage of $70 \%$ would have reduced the estimate of vaccine effectiveness to $73 \%$. Age and place of admission may also have confounded the estimate as we did not stratify the analyses according to these variables (8). The presence of measles transmission in spite of a high estimate of vaccine effectiveness in this study, points to possible gaps in actual vaccination reach to susceptible groups in Nairobi which the immunisation programme should address. As the general immunisation coverage in Nairobi was $93 \%$ in 1998 (22), the problem cannot be in the whole city but may be confined to certain groups such as those in highly densely populated slums.

This review showed high case fatality rates in 1996, 1998 and 1999 in settings where good clinical management would be expected. Possible explanations may be given for the unusually high mortality from measles in these two hospitals. The most plausible explanation was that the majority of cases presented late when symptoms were already complicated and severe. The potential contribution by the build up of government nurses' strike in 1997 and its carry over effect towards increased case fatalities cannot be ruled out. Another possible explanation for the high case fatality rate in 1998 may have had to do with the presentation of more secondary cases with more severe symptoms as there was an outbreak in that year.

\section{REFERENCES}

1. Centers for Disease Control. Progress towards global measles control and elimination, 1990-1996. Morb. Mortality Weekly Review. 1997; 46:893-897.

2. Expanded programme on Immunisation. Global measles mortality and regional elimination. Weekly Epidemiol. Record. 2002; 77:51-54.

3. Cutts, F.T., Henderson R., Clements C.J., et al. Principles of measles control. Bulletin World Health Org. 1991b; 69:1-7.

4. Chen, R.T., Weierbach R., Bisoffi. Z., et al. 'post-honeymoon period' measles outbreaks in Muyinga sector, Burundi. International J. Epid. 1994; 23:185-193.

5. McLean, A.R., and Anderson, R.M. Measles in developing countries. II. The predicted impact of mass immunisation. Epidemiology and Infection. 1988; 100:419-442.

6. Begg N., and Miller E. Role of epidemiology in policy. Vaccine. 1990; 8:180-189

7. Halloran, M.E., Struchiner, C.J. and Longini, I.M. Study designs for evaluating different efficacy and effectiveness aspects of vaccines. Amer. J. Epid. 1997; 146:789-803.

8. Farrington, C.P. Estimation of vaccine effectiveness using the screening method. International J. Epid. 1993; 22:742-746.

9. Centers for Disease Control. Pertussis - United States, 19972000. Morb. Mortal. Weekly Review. 2002; 51:73-76.

10. Guris, D., Strebbel, P.M., Tachdjian R., et al. Effectiveness of the pertusis vaccination program as determined by use of the screening method: United States, 1992-1994. J. Infectious Dis. 1997; 176: 456-463.

11. Hatton, $\mathrm{P}$. The use of the screening technique as a method of rapidly estimating vaccine effectiveness. Public Health. 1990; 104:21-25

12. Burstrom, B., Aaby P. and Mutie D. Child mortality impact of a measles outbreak in a partially vaccinated rural African community. Scandinavian J. Infectious Dis. 1993; 25:763-769. 
13. Burstrom, B., Aaby, P., Mutie, D.M., Kimani, G. and Bjerregaard, P. Severe measles outbreak in western Kenya. East Afr. Med. J. 1992; 69:419-423.

14. Kenya, P.R. Measles and mathematics: control or eradication? East Afr. Med. J. 1990; 67:856-863.

15. Stata Corporation. STATA statistical software. Stata Corporation 4905 Lakeway Drive College Station, Texas 77845 USA.

16. Cutts, F.T., Othepa, O., Vernon, A.A., et al. Measles control in Kinshasa, Zaire. Improved with high coverage and use of medium titre Edmonston Zagreb vaccine at 6 months. International J. Epid. 1994; 23:624-631.

17. Hayden, R.J. The epidemiology and nature of measles in Nairobi before the impact of measles immunisations. East Afr. Med. J. 1974; 51:199-205

18. Taylor, W.R. Ruti-Kalisa, ma-Disu, M., and Weinman J.M. Measles control efforts in urban Africa complicated high incidence of measles in the first year of life. Amer. J. Epid. 1988; 127:788-794.

19. Luthi, J.C., Kessler, W. and Boelaert, M. Une enquete d'efficite vaccinale dans la ville de Bongor (Tchad) et ses consequences operationneles pour le programme de vaccination. Bulletin World Health Org. 1997; 75:427-433.

20. Mgone, J.M., Mgone, C.S., Duke, T., Frank, D., and Yeka, W. Control measures and the outcome of the measles epidemic of 1999 in Eastern Highlands Province. Papua New Guinea Med. J. 2000; 43:91-97.

21. Kambarani, R.A., Nathoo, K.J., Nkrumah, F.K. and Pirie, D.J. Measles epidemic in Harare, Zimbabwe, despite high measles immunisation coverage rates. Bulletin World Health Org. 1991; 69:213-219.

22. Central Bureau of Statistics (Ministry of Finance and Planning GOK). Vaccinations. The Kenya Health and Demography Survey. 1998; 104-106. 